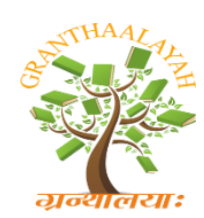

\author{
INTERNATIONAL JOURNAL OF RESEARCH - \\ GRANTHAALAYAH \\ A knowledge Repository
}

Science

\title{
A STUDY OF RADIO FREQUENCY BLACKOUT FOR SPACE PROBE DURING ATMOSPHERIC REENTRY PHASE
}

\author{
Sidi Ahmed Bendoukha ${ }^{* 1}$, Kei-ichi Okuyama ${ }^{2}$, Bianca Szasz ${ }^{3}$ \\ *1, 2, 3 Applied Sciences and Integrated System Engineering, Kyushu Institute of Technology, 1-1 \\ Sensui-cho, Tobata Ward, Kitakyushu, Fukuoka Prefecture 804-0015, Japan
}

DOI: https://doi.org/10.29121/granthaalayah.v5.i3.2017.1750

\begin{abstract}
During the re-entry flight, the radio signal will be interrupted, which is commonly referred to as the communications blackout. Once the plasma sheath forms in the stagnation region of a small space probe, the probe losses more than 70 percent of its downlink data. This shows that the attenuation of the radio signal is very high during the re-entry. When the probe enters the Earth's atmosphere, the high velocity, high surface temperature and high plasma frequency cause a shock wave layer, which is the main cause of radio blackout. For other reason, the completely reflection of the electromagnetic wave at all communication lines. This study describes the theoretical and numerical study of radio communication during reentry. The paper defines an approach to end radio signal blackout occurring in the wake region and how to exactly solve the radio blackout problem using new methods as injection of coolants, the aerodynamic shaping reducing the concentration of electrons, using transceiver with high operating frequency or interaction of Static Magnetic Field (SMF). Data from OREX probe are used to prove the solution to the Radio Frequency (RF) blackout problem. The significance of the used SMF method is established by computing the reduction in plasma attenuation.
\end{abstract}

Keywords: Radio Frequency; Plasma Density; Communication Blackout; Collision Frequency; Attenuation Signal; Magnetic Field; Reentry.

Cite This Article: Sidi Ahmed Bendoukha, Kei-ichi Okuyama, and Bianca Szasz. (2017). “A STUDY OF RADIO FREQUENCY BLACKOUT FOR SPACE PROBE DURING ATMOSPHERIC REENTRY PHASE." International Journal of Research Granthaalayah, 5(3), 1-15. https://doi.org/10.29121/granthaalayah.v5.i3.2017.1750.

\section{Introduction}

The Orbital Re-entry Experiments (OREX) space probe was launched in February 1994 using an $\mathrm{H}-\mathrm{II}$ rocket that lifted off from the Tanegashima Space Center in Japan [1], and later re-entered the Earth's atmosphere. Re-entry was a critical epoch when all communications between the space probe and ground stations could not be established. This occurred because of several 
factors such as high heat flux, high speed and the plasma sheath forming during re-entry; this phenomenon is usually known as a communication blackout.

In addition, when high operation frequency radio waves pass through the Earth's atmosphere, the signals also experiences attenuation and phase reflection of the propagating wave. The signal degradation mostly takes place in the lower part of plasma formation. The space probe was exposed to this shock because its velocity was much greater than the speed of sound, thus a shock layer formed in the front of the probe. The plasma flow shaped from thermal ionization of the gas as it is compressed and heated within the boundary of the surface temperature. Because the electron plasma density surrounding the probe is very high, communications are interrupted during the entry phase defined as radio frequency RF cut-off [2] shown in Fig. 1.

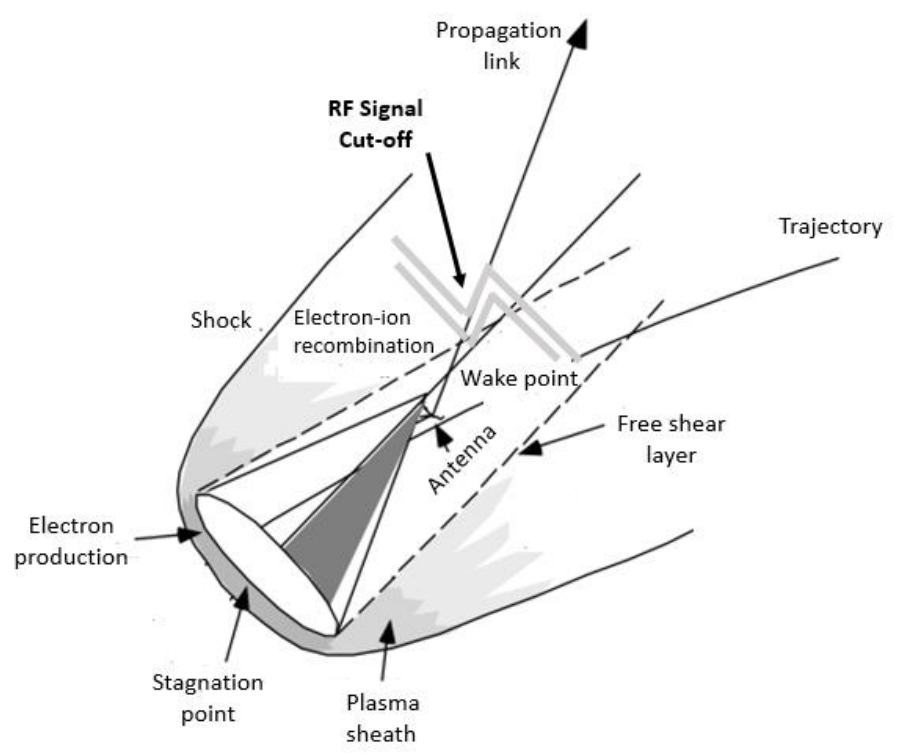

Figure 1: Space probe atmospheric entry.

The paper defines the theoretical concept, analysis for plasma formation and numerical simulations for communication blackout. A typically probe reentry of Fig. 2, showing the communication blackout area in function of reentry time (time before touchdown) [3].

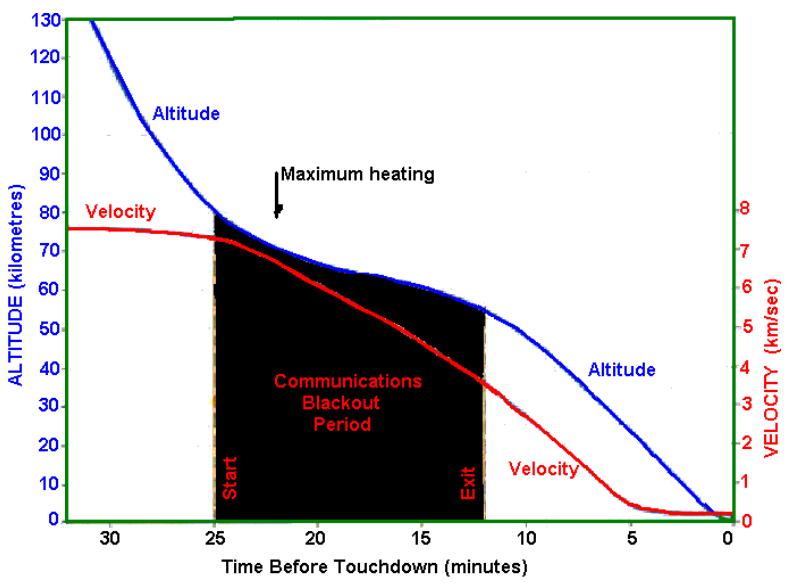

Figure 2: Typical space probe RF blackout reentry. 
The current work focuses on calculation of attenuation signal and phase by varying the operating frequency, variation of the ratio between the collision frequency and plasma frequency. Furthermore, methods to overcome the RF blackout, such as injection of coolants, aerodynamic shaping that reduces the concentration of electrons density, or using transceiver with high frequency of operation, are briefly illustrated and evaluated here. The most promising methods are: use a precise technique, such as interaction of SMF windows with plasma sheath to reduce the RF attenuation and its impact reentry. An examination has been done for a typical reentry probe, to resolve the propagation link between the space probe and targets.

\section{Space Probe Reentry Simulations}

When a space probe such as OREX or the space shuttle leaves orbit and reenters to the atmosphere, it typically travels to a landing site. In this section, a brief explanation and simulations using OREX data and measured data at Japan Aerospace Exploration Agency/Institute of Space and Aeronautical Sciences (JAXA/ISAS) for reentry blackout using LATS [4] material as thermal shield, are used to demonstrate the reentry phase. In low altitude orbit of the space probe or alike vehicle is travelling at high velocity about $7.8(\mathrm{~km} / \mathrm{s})$. During this phase, the electron density is very high and forms a plasma sheath. The 2D plot of Fig. 3 clarifies the relationship between the variation of plasma density, velocity and altitude. The velocity fluctuates little due to an exchange of gravitational potential energy. At nearby $100 \mathrm{~km}$ altitude, the atmospheric drag increases and the resulting heat flux (more than $3 \mathrm{MW} / \mathrm{m} 3$ for OREX) increases such as the energy is swapped to thermal energy providing the plasma flow in the thermal shield of probe. The 2D plots in 3 axis of Fig. 4 and Fig. 5 illustrate the relationship between the variation of velocity, pressure and altitude.

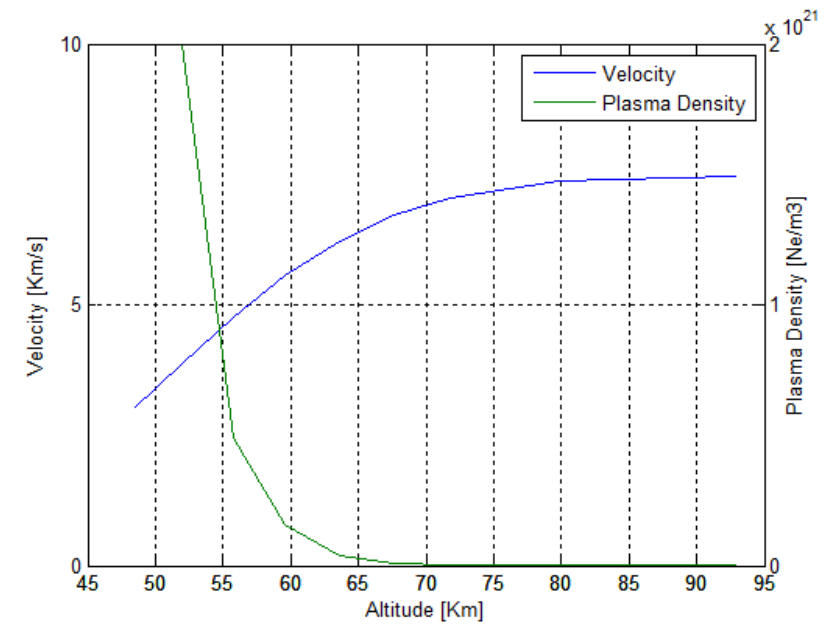

Figure 3: Plot of variation of velocity in function the electrons density 


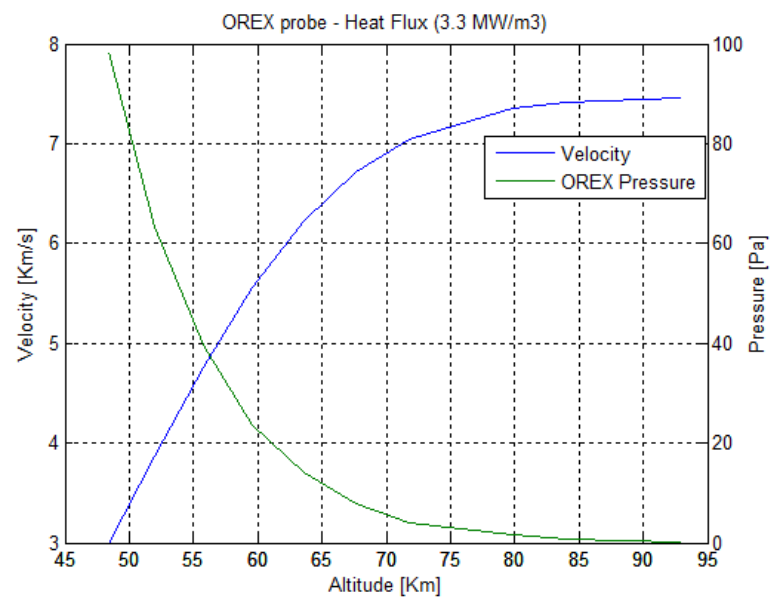

Figure 4: Variation of velocity and the pressure in function of the altitude for OREX probe

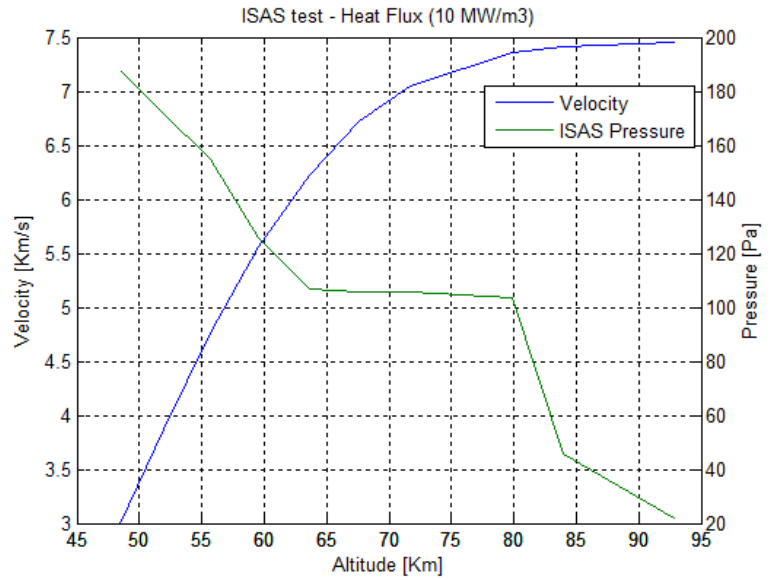

Figure 5: Variation of velocity and pressure in function of the altitude for ISAS test

The Fig. 6 shows the variation of surface temperature (wall temperature) of OREX probe in function of orbit altitude during the reentry.

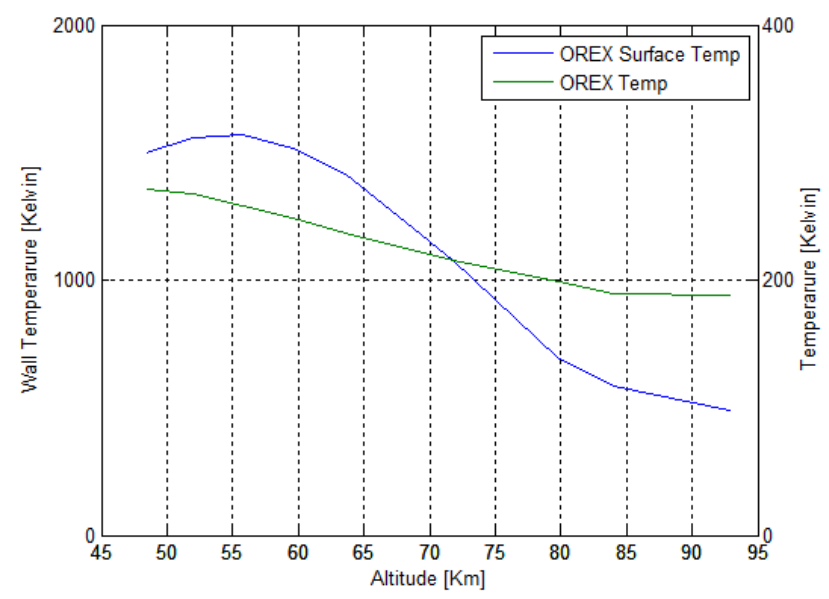

Figure 6: Variation of temperature in function the altitude for OREX probe 


\section{Methodology \& Solution}

\subsection{Plasma Sheath and Propagation Link}

Once the plasma sheath occurred in stagnation region of the space probe showed in Fig. 1. A major outcome of electrons in the plasma is to allow the absorption of kinetic energy from the electromagnetic wave. This frequency is called angular plasma frequency and can be calculated as:

$$
f_{p}=\frac{1}{2 \pi}\left(\frac{N_{e} q_{e}^{2}}{£ M_{e}}\right)^{\frac{1}{2}}
$$

Where the $f_{p}(\mathrm{GHz})$ is the plasma frequency, $£$ the dielectric constant in free space, $N_{e}\left(\mathrm{~cm}^{-3}\right)$ is the number of electron density and $\left(M_{e}, q_{e}\right)$ are the mass of electron, elementary charge. By expanding the constants, the plasma frequency can be written as:

$$
f_{p}=9 \times 10^{-6}\left(N_{e}\right)^{\frac{1}{2}}
$$

When the electrons undulations were raised in the plasma sheath, an electron and ion of plasma flow can collide through several unbiased particles. This frequency known as collision frequency $\left(\right.$ Coll $\left._{f}\right)$, is the amount of collided particles per second that the electrons across with other sorts in the plasma, Therefore, would determine the amount in $\mathrm{dB} / \mathrm{m}(\mathrm{Neper} / \mathrm{m})$ of attenuation. Once the frequency of operation was higher than the plasma frequency. The collision plasma can be dependent by surface temperature $(\mathrm{T})$ and pressure $(\mathrm{P})$ in specific point of stagnation region, using the basic laws of the plasma conductivity [5]. The collision plasma is written as:

$$
\operatorname{Coll}_{f}=\frac{58.2 \times 10^{11} P}{\sqrt{T}}
$$

Various measured data of the OREX probe, ISAS tests, and theoretical data were compared for calculation of collision plasma frequency shown in Fig.7, Fig. 8 and Fig. 9.

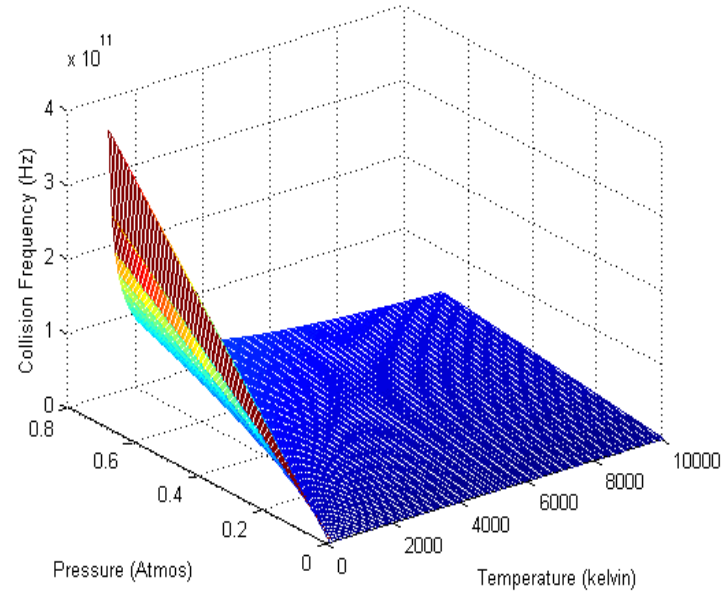

Figure 7: Collision Plasma for typical probe 


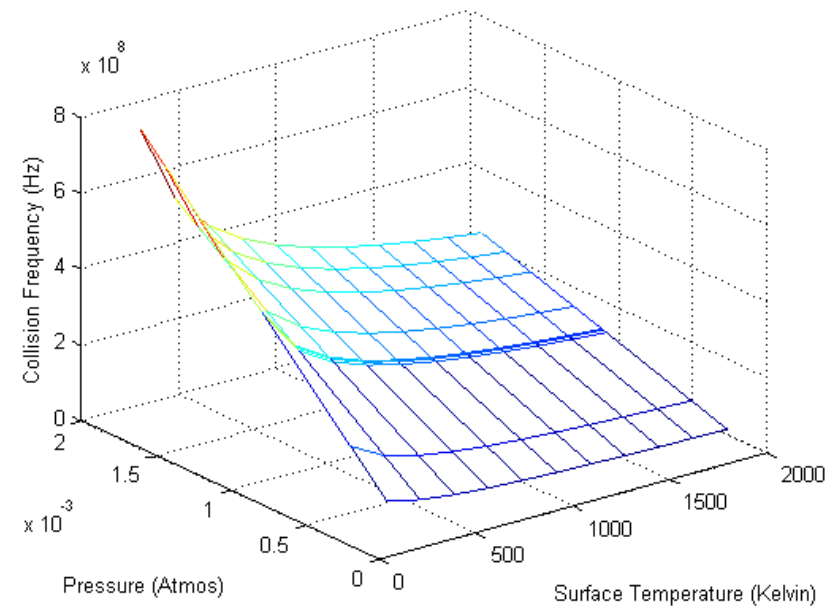

Figure 8: Collision plasma for ISAS data

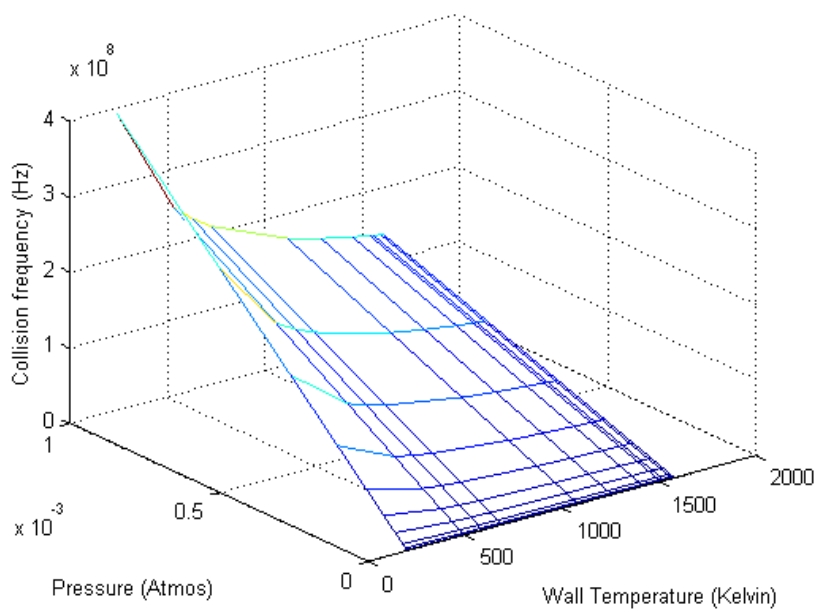

Figure 9: Collision plasma of OREX probe

\subsection{Attenuation \& Phase of Electromagnetic Propagation Wave}

The RF blackout communication caused by the effects of charged particles in the surrounding plasma on a space probe reentry will attenuate any transmission wave by reflection and absorption of electromagnetic waves. The charged particles (electrons) are the core suppliers to reflect the RF signal in the wall of probe. The wave propagation coefficients (real and imaginary) were expressed by wave quantity of free space (speed of light), collision frequency, plasma frequency and operating frequency $(f)$ of the plasma flow. Table 1 describes the critical electron densities for space probe reentry based on the frequency allocation.

Table 1: Critical values of electrons densities

\begin{tabular}{lll}
\hline Plasma Densities $\left(\mathbf{c m}^{-\mathbf{3}}\right)$ & \multicolumn{1}{|c}{ Operating Frequency $(\mathbf{G H z})$} & Frequency Allocation Band \\
$2.59 \times 10^{8}$ & 0.145 & Very High Frequency VHF \\
$2.36 \times 10^{9}$ & 0.437 & Ultra-High Frequency UHF \\
$3.06 \times 10^{10}$ & 1.575 & L1 GPS positioning \\
\hline
\end{tabular}




\begin{tabular}{lll}
\hline $7.11 \times 10^{10}$ & 2.4 & S-band \\
$8.30 \times 10^{11}$ & 8.2 & X-band \\
$1.28 \times 10^{13}$ & 32.2 & Ka band \\
\hline
\end{tabular}

Several techniques were used in the past as integration of electromagnetic field with plasma sheath. This method was applied in this paper. Therefore, the plasma frequency is determined by the electrical conductivities and dielectric constant deviate atop the frequency allocation. The electromagnetic wave is propagated in wake region can be specified by attenuation $(x)$ and phase $(y)$ coefficients defined in a complex formula as follow [6]:

$$
\delta=x-j y
$$

Where $\delta$ is the complex propagating wave (the complex relative permittivity). The relative permittivity $\left(\varepsilon_{p}\right)$ and dielectric conductivity $\left(\varepsilon_{d}\right)$ of the complex number are given (5) and (6). Those parameters are simplified as function $(\varnothing)$ as follow:

$$
\begin{aligned}
& x=\emptyset\left[\varepsilon_{p}, \varepsilon_{d}, K\right] \\
& y=\emptyset\left[\varepsilon_{p}, \varepsilon_{d}, K\right]
\end{aligned}
$$

Where: $K=w / c,(c)$ is speed of light and $(w)$ is angular frequency. The variables $\left(\varepsilon_{p}\right)$ and $\left(\varepsilon_{d}\right)$ are dependent to plasma frequency and frequency range, are expressed as:

$$
\begin{gathered}
\varepsilon_{p}=1-\frac{1}{\alpha^{2}+\left(\frac{f}{f_{p}}\right)^{2}} \\
\varepsilon_{d}=\frac{\alpha \times\left(\frac{f_{p}}{f}\right)}{\alpha^{2}+\left(\frac{f}{f_{p}}\right)^{2}}
\end{gathered}
$$

Where: $\alpha=\frac{\operatorname{coll}_{f}}{f_{p}}$ is the ratio between the collision frequency and plasma frequency.

If collision frequencies are neglected, the dielectric conductivity $\left(\varepsilon_{d}\right)$ became zero, and the relative permittivity $\varepsilon_{p}$ assumed as:

$$
\varepsilon_{p}=1-\left(\frac{f_{p}}{f}\right)^{2}
$$

The attenuation $(\mathrm{Neper} / \mathrm{m}=8.65 \mathrm{~dB} / \mathrm{m})$ and phase shift $(\mathrm{Deg} / \mathrm{m}=180 / \mathrm{pi} \mathrm{dB} / \mathrm{m})$ coefficients are written as follow:

$$
\begin{aligned}
& x^{2}=\frac{K^{2}}{2}\left[\left(\varepsilon_{p}^{2}+\varepsilon_{d}^{2}\right)^{\frac{1}{2}}-\varepsilon_{p}\right] \\
& y^{2}=\frac{K^{2}}{2}\left[\left(\varepsilon_{p}{ }^{2}+\varepsilon_{d}^{2}\right)^{\frac{1}{2}}+\varepsilon_{p}\right]
\end{aligned}
$$


In case of collision frequency [7] less plasma frequency ( Coll $_{f}$ neglected), the attenuation coefficient $(x)$ drops to zero and the phase coefficient $(y)$ is assumed to be:

$$
y=K \sqrt{1-\left(\frac{f_{p}}{f}\right)^{2}}
$$

Table 2 illustrates the description of the communication blackout during reentry.

Table 2: Description of RF blackout

\begin{tabular}{ll}
\hline Frequency Range & Clarifications \\
$f>f_{p}$ & Communication established (RF signal across the plasma sheath) \\
$f=f_{p}$ & Phase coefficient became zero (RF signal completely reflected) \\
$f<f_{p}$ & Communication blackout (no penetration of RF signal) \\
\hline
\end{tabular}

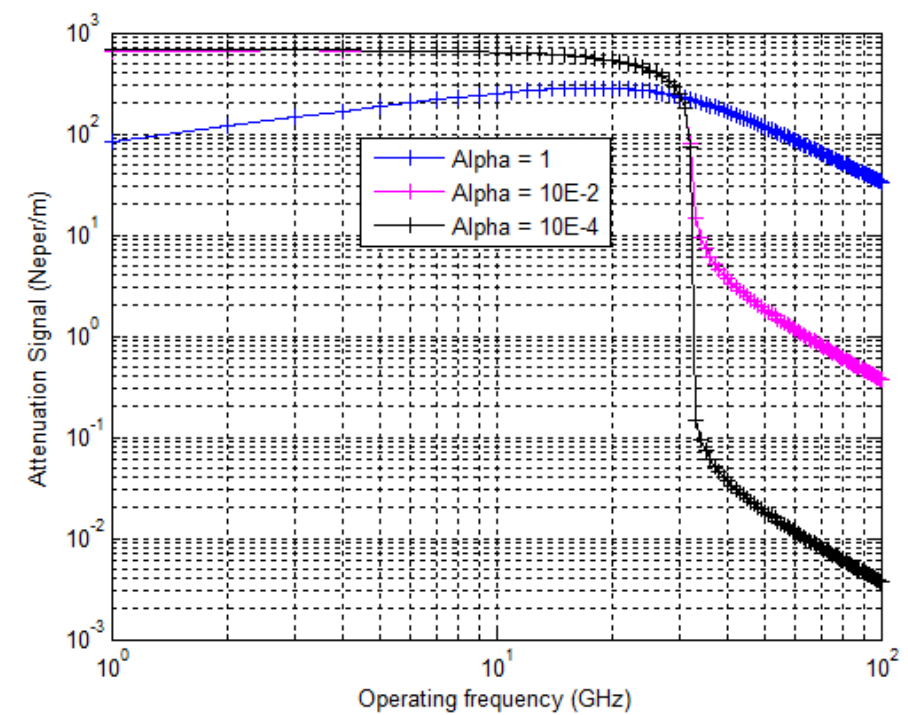

Figure 10: Attenuation signal $\left(N_{e}=1.28 \times 10^{13} \mathrm{~cm}^{-3}\right)$

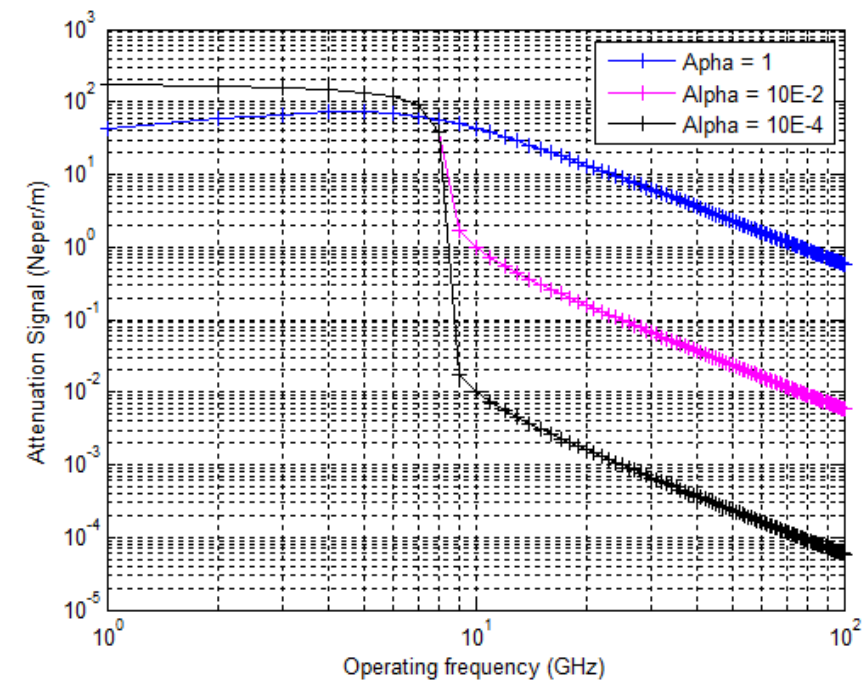

Figure 11: Attenuation signal $\left(N_{e}=8.30 \times 10^{11} \mathrm{~cm}^{-3}\right)$ 
In the Fig. 10 and Fig.11, the selected plasma frequencies are equal to Ka carrier frequency $(32 \mathrm{GHz})$ and $\mathrm{X}$ carrier frequency $(8 \mathrm{GHz})$ dependent to the electron densities values shown in figures above. When the ratio $(\alpha)$ inreceases (the attenuation higher), the transmission signal is temporarily interrupted which is named RF blackout cut-off. Therefore, for other case of the operating frequency is higher then plasma frequency (the ratio $(\alpha)$ lower), the transmission of waves can propagate through the plasma sheath. When the $\alpha=1$, the $\left(N_{e}\right)$ known as crirical density.

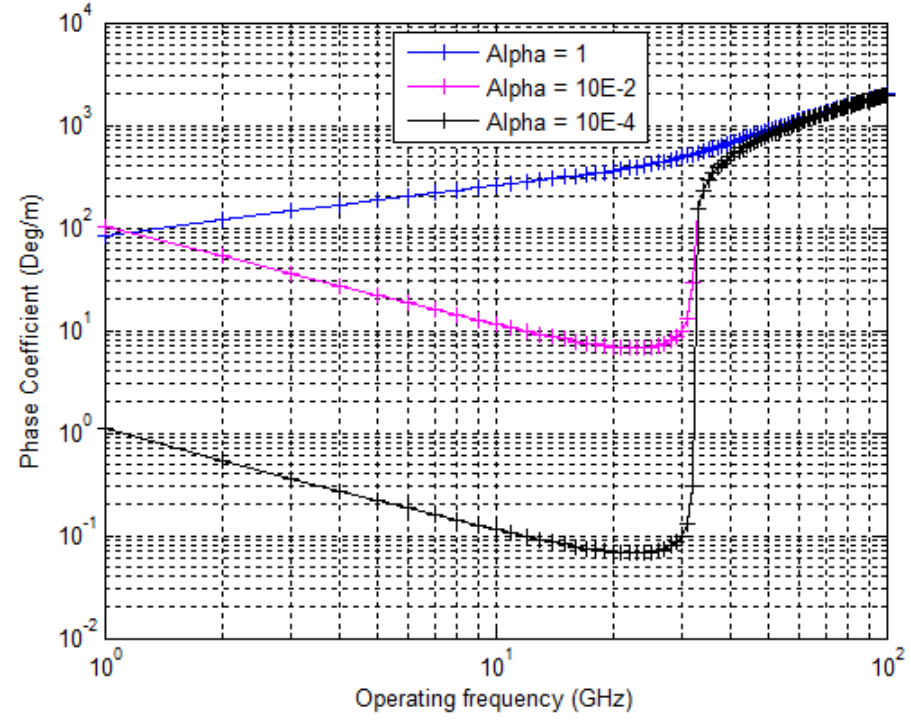

Figure 12: Phase coefficient $\left(N_{e}=1.28 \times 10^{13} \mathrm{~cm}^{-3}\right)$

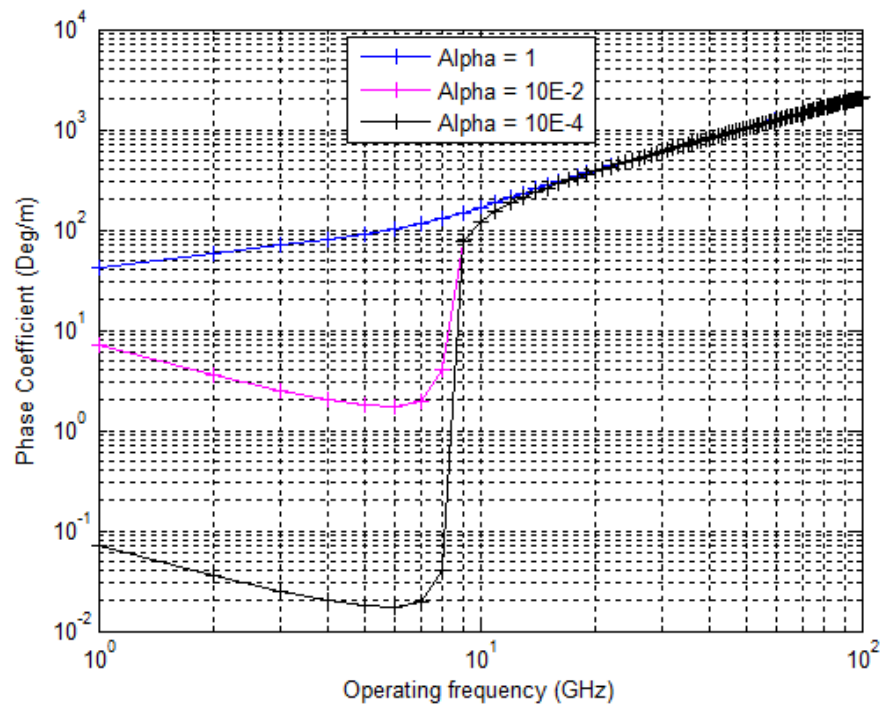

Figure 13: Phase coefficient $\left(N_{e}=8.30 \times 10^{11} \mathrm{~cm}^{-3}\right)$

In the Fig. 12 and Fig.13, the selected plasma frequency values are the same as attenuation curves. Once the ratio $(\alpha)$ decreases, the reflection coefficient reduces also (communication establsihed). When the operating frequency is higher then the plasma frequency, the phase coeficient strictly became linear and independent to collosion frequency. 


\subsection{Approaches for Solving Communications RF Blackout}

Since some years ago, numerous techniques were in development to resolve the RF blackout cutoff difficulty to during earth's atmospheric re-entry. The recommended [8] approaches are as follows:

- Aerodynamic shaping method known as remote antenna assemblies

- Raman scattering process (electromagnetic pump wave with high frequency)

- Injection of quenchants (liquid/gas injection)

- SMF windows (interaction of electromagnetic filed in the plasma sheath)

- Using space probe transceiver with upper frequency carrier

One of those methods is illustrated in this section, the SMF window mitigation is used in this paper to figure out the problem of communication reentry and evaluated in terms of realistic applications, means to achieve the minimum amount of plasma electron densities (lower attenuation) dependent to variation of magnetic field strength.

\subsubsection{SMF Approach}

This paper presents the impact of static magnetic field on the wake region near to the antenna shown in Fig. 14. The outcomes designate that the influence of the ion-electron recombination of the plasma density on the antenna performance can be reduced when the permanent magnet intensity increased. This approach can solve this problem in a direction perpendicular to the incident of the propagating wave. In the schematic in Fig. 14, the RF signal is reflected without implementing SMF. Hence, SMF integrated to the plasma wall, a window is created in this region allowed to establish RF transmission link at inferior frequency allocation bands.
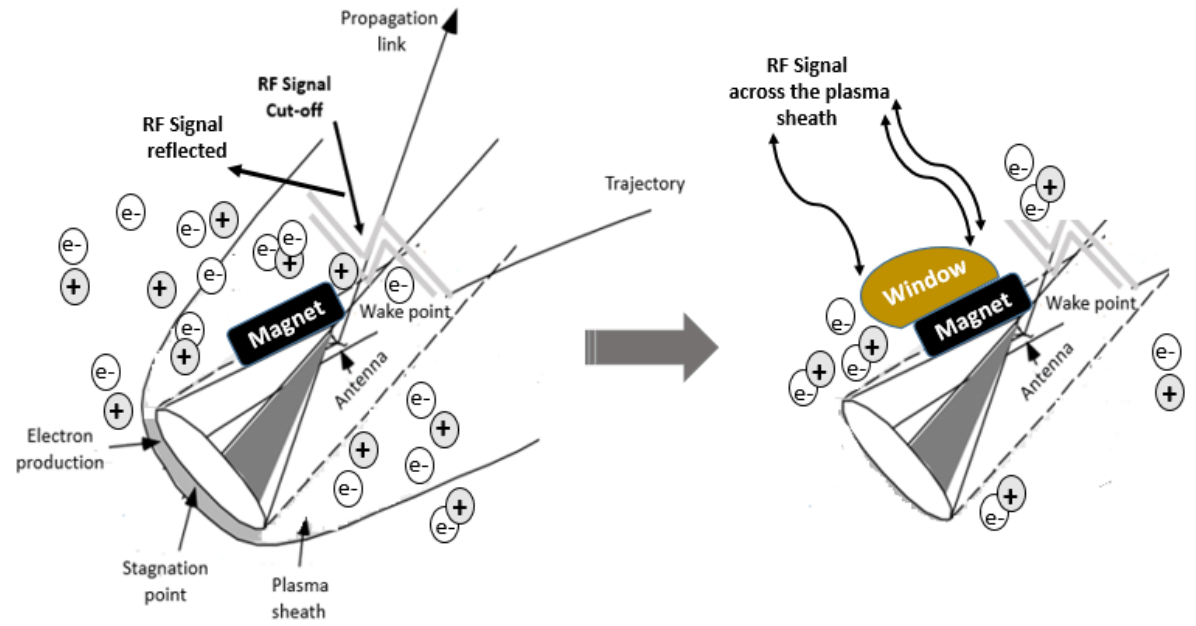

Figure 14: A Schematic of space probe including permanent magnet for mitigation method

The complex number defined in (4) of the magnetized plasma if the SMF direction is in the same direction of the propagation link dependent the polarization directions [9], is written as: 


$$
\delta=1-\frac{f_{p}^{2}}{f^{2}\left[\left(1-j \frac{\operatorname{Coll}_{f}}{2 \pi f}\right) \pm \frac{f_{o s c}}{f}\right]}
$$

Where $f_{\text {osc }}$ is the oscillating frequency or cyclotron frequency during the circular motion of charged particles in plasma sheath.

$$
S M F_{\text {min_thres }}=2 \pi \frac{M_{e}}{q_{e}} f_{\text {osc }}
$$

$S M F_{\text {min_thres }}$ is the minimum threshold of the magnetic field strength that can be applied in the plasma sheath of the space probe. The oscillation frequency can be simplified as:

$$
f_{\text {osc }}(\mathrm{GHz})=27.98 \times S M F_{\text {min_thres }}
$$

This paper describes modeling and simulations for the method used to reduce the attenuation coefficient by varying strength of the magnet and to define the minumum threshold amount of magnetic field intensity applied for diffrent frequencies carrier ( $\mathrm{Ka}$ and $\mathrm{X}$ bands) dependent on the plasma frequency and collision frequency. Therefore, the actual value required of magnetic field strength in practical cases should be higher than $S M F_{\text {min_thres }}$. For Ka band, the required SMF strength should be more than 1.1Tesla and for X band is greater than 0.3Tesla. The Table 3 illlustrates the applied SMF in the propagation link.

Table 3: Threshold magnetic field strength for communication blackout

\begin{tabular}{ll}
\hline SMF Range & Clarifications \\
$S M F_{\text {min_thres }}>S M F$ & RF signal through the plasma sheath, the communication established \\
$S M F_{\text {min_thres }}<S M F$ & RF signal totally reflected, communication blackout occurred \\
\hline
\end{tabular}

The attenuation and reflection coefficients using the SMF method by varying the collision frequency are plotted using (13), are shown in Fig. 15 to Fig. 18.

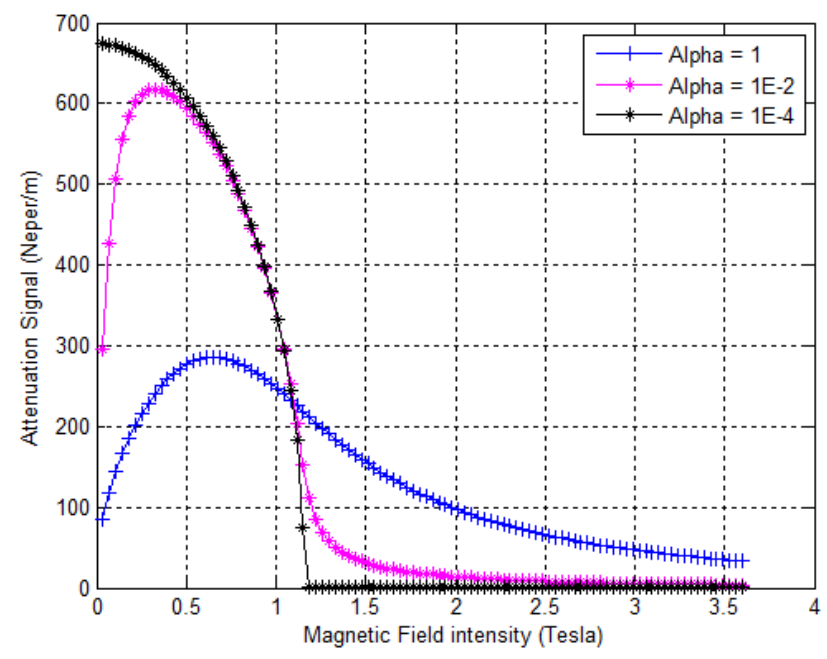

Figure 15: Attenuation signal versus magnetic field strength (Ka band) 


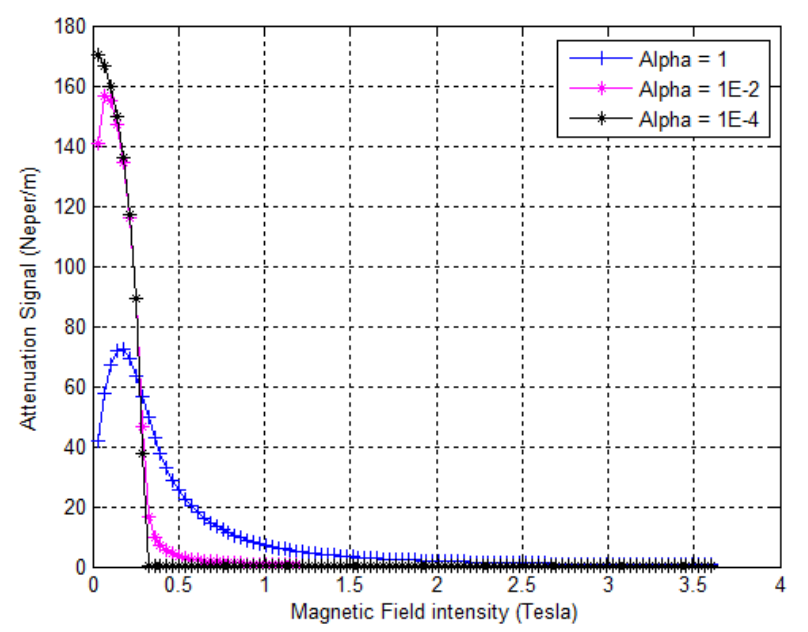

Figure 16: Attenuation signal versus magnetic field strength ( $\mathrm{X}$ band)

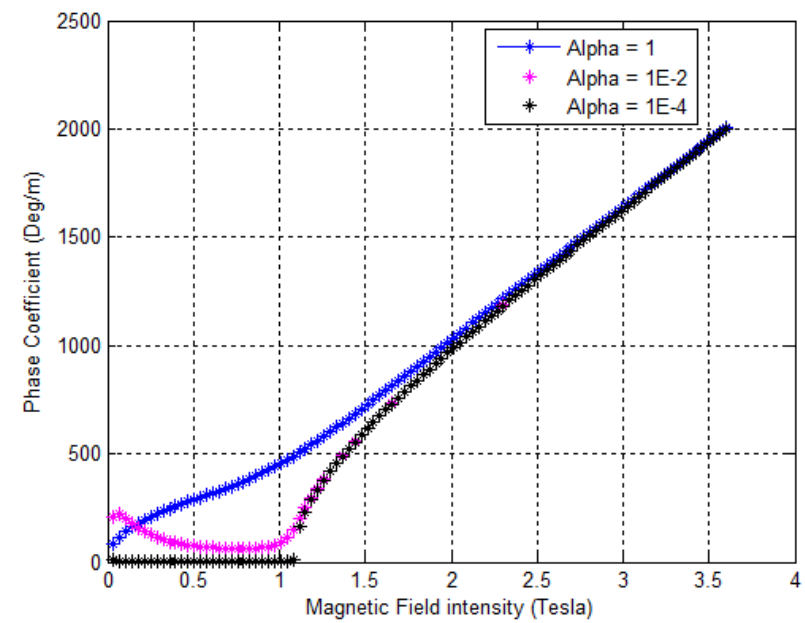

Figure 17: Phase shift versus magnetic field strength (Ka band)

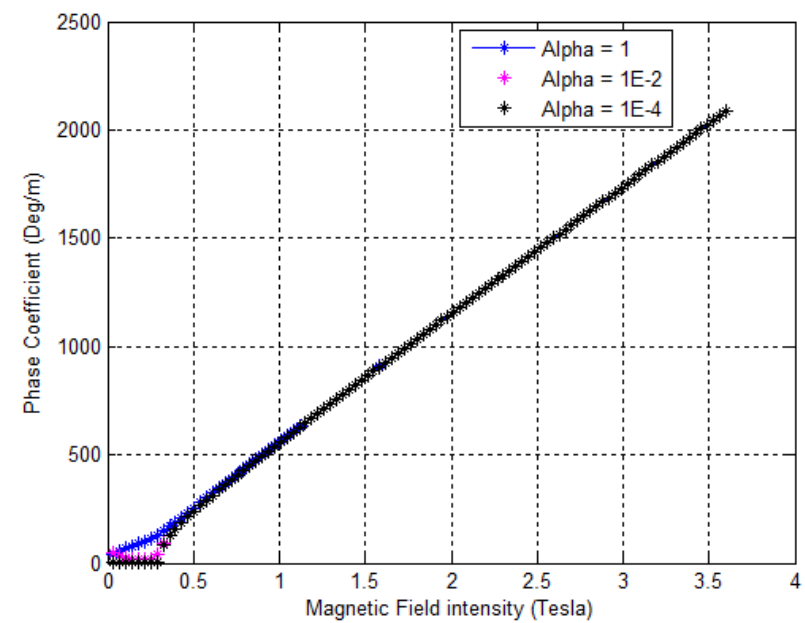

Figure 18: Phase shift versus magnetic field strength ( $\mathrm{X}$ band) 
The Fig. 19 illustrates the attenuation signal $(\mathrm{dB} / \mathrm{m})$ in function the minimum magnetic field for all frequencies bands when the electron collision frequency is equal to the plasma angular frequency $(\alpha=1)$.

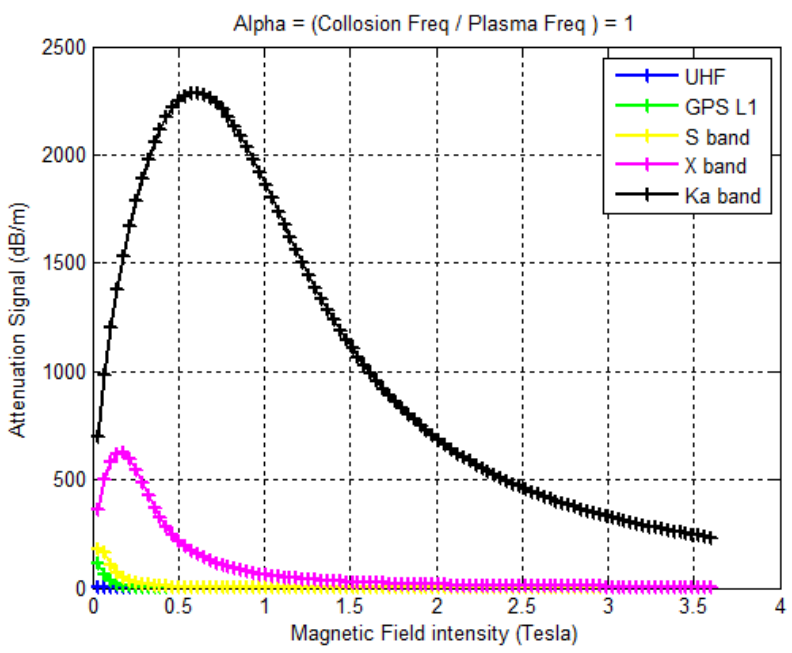

Figure 19: Minumum magnetic field strength for frequencies allocation

Furthermore, the permanent magnetic of external magnetic field which reduces the attenuation of the transmitting wave in plasma to $31 \mathrm{~dB}$. The attenuation was about $81 \mathrm{~dB}$ ( $700 \mathrm{Neper}$ ) for Ka band defined in Fig. 10 comparing the Fig. 20 at electron density about $N_{e}=1.28 \times 10^{13} \mathrm{~cm}^{-3}$ (Ka band) the attenuation decrease to $50 \mathrm{~dB}$. It needs to increase more the SMF strength to reach the minimum value of plasma density, it's allowed to communicate in the wake region of the plasma flow.

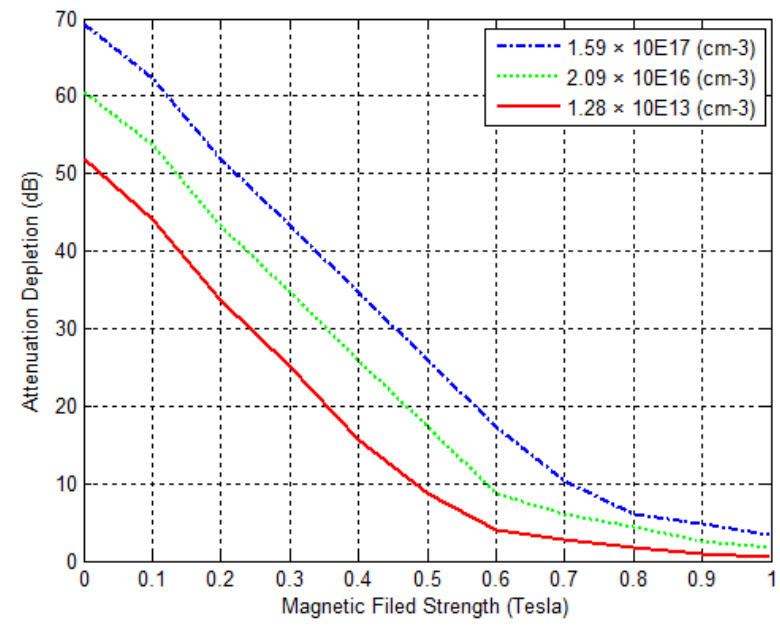

Figure 20: Attenuation reduction with SMF

\section{Conclusions \& Recommendations}

The paper concludes the suggest SMF method used for solving the communication problem of Earth's atmospheric re-entry of space probe. Numerical simulations and analysis were conducted 
to prove the cause of the RF blackout and how to integrate the magnet field with electromagnetic wave surrounding the plasma sheath. Various measured data and theoretical were compared for calculation of collision frequency in function of pressure and surface temperature. The SMF technique was developed in this work to reduce the electrons plasma densities by varying the magnet field strength for different frequencies carriers. When the permanent magnet installed in the space probe, a windows was created to be easy for RF signal to across the plasma sheath (communication established). The goal of this study is to present the results of reduction of attenuation of the electromagnetic wave and defines the minimum threshold magnetic field intensity in the communication region. For the future, this study can be helpful for the researchers to develop in deeply the problem of communication blackout and implement new techniques to reduce the plasma frequency.

\section{Acknowledgements}

This study was held in Kyushu Institute of Technology of Japan, especially at Okuyama laboratory. I would like to thank the UNOOSA United Nations Office Outer Space Affairs that offer to me Japan Long-Term Fellowship Program 2014 until 2017. The present computational results were obtained and simulated using the facilities of Japan Aerospace Exploration Agency (JAXA). I'm also grateful to my fellow doctoral and master students for their feedback, cooperation and of course friendship. In addition, many thanks to Professor and my appreciations to all who helped and oriented me.

\section{References}

[1] Yukimitsu Yamamoto. "RECENT COMPARISONS OF AEROTHERMODYNAMICS RESULTS BY CFD AND FEM COUPLING ANALYSIS WITH OREX FLIGHT EXPERIMENTS, JAXA.” National Aerospace Laboratory 1996-01, Japan.

[2] Yusuke Takahashi, Reo Nakasato and Nobuyuki Oshima. "ANALYSIS OF RADIO FREQUENCY BLACKOUT FOR A BLUNT-BODY CAPSULE IN ATMOSPHERIC REENTRY MISSIONS.” Aerospace 2016, 3(1), 2; doi:10.3390/aerospace3010002.

[3] Hannah Camelotti, RE- ENTRY AND SAFE LANDING OF A SPACE SHUTTLE SPACECRAFT REENTRY COMMUNICATIONS BLACKOUT, Australian Space Academy, October 2014, Science.

[4] Sidi Ahmed Bendoukha, Kei-ichi Okuyama, Bianca Szasz, Takayuki Shinoda. "EXPERIMENTAL METHOD USING ARC WIND TUNNEL TO SIMULATE A NEW TECHNIQUE FOR COMMUNICATING WITH A REENTRY PROBE HAVING A THERMAL SHIELD MADE OF LATS MATERIALS." International Journal of ResearchGRANTHAALAYAH, Vol. 5, Iss.2. 02, 2017; doi.org/10.5281/zenodo.345434.

[5] Jiamin Chen, Kai Yuan, Linfang Shen, Xiaohua Deng, Lujun Hong, and Ming YaoLankford, "STUDIES OF TERAHERTZ WAVE PROPAGATION IN REALISTIC REENTRY PLASMA SHEATH.” Progress in Electromagnetics Research, Vol. 157, 21-29, 2016.

[6] Hodara, H., "THE USE OF MAGNETIC FIELDS IN THE ELIMINATION OF THE REENTRY RADIO BLACKOUT.’Proceedings of the IRE, Vol. 49, No. 12, 1825-1830, 1961.

[7] Donald S.Frankel, Peter E, Nebolsine, Merlin G.Miller, James M. Glynn. "A REENTRY PLASMA INDUCTED PSEUDORANGE AND ATTNUATION EFFECTS IN A GPS SIMULATOR,” PSI-SR-1175. April 2004. 
[8] Kim, M., M. Keidar, and I. D. Boyd, "ANALYSIS OF AN ELECTROMAGNETIC MITIGATION SCHEME FOR REENTRY," Journal of Spacecraft and Rockets, Vol. 45, Iss. 6, Nov 2008,

[9] Xiao Jun Xing, Qing Zhao and Ling Zheng. "THEORETICAL AND EXPERIMENTAL STUDIES OF MAGNETIC FIELD ON ELECTROMAGNETIC WAVE PROPAGATION IN PLASMA.” Progress in Electromagnetics Research M, Vol. 30, 129-139, 2013.

*Corresponding author.

E-mail address: 0595904a@ mail.kyutech.jp 